\title{
Exploring the $\gamma^{*} p \rightarrow \Delta(1232)$ Transition at Low $Q^{2}$ Using CLAS
}

\author{
L.C. Smith \\ (for the CLAS Collaboration) \\ Physics Department, University of Virginia, Charlottesville, Va. 22901
}

\begin{abstract}
Precision measurements of the $p\left(e, e^{t} p\right) \pi^{0}$ reaction were performed using the CEBAF Large Acceptance Spectrometer (CLAS) in the range of photon invariant momentum transfer $Q^{2}=0.16-0.36 \mathrm{GeV}^{2}$. Fits to the data using both a Unitary Isobar Model and the Sato-Lee dynamical model are used to estimate the 'dressed' and 'bare' electromagnetic couplings for the magnetic and quadrupole $\gamma^{*} p \rightarrow \Delta(1232)$ transition form factors.
\end{abstract}

Keywords: Resonance, Delta, Deformation, Pion

PACS: $13.60 . \mathrm{Le}, 13.40 . \mathrm{Gp}, 14.20 . \mathrm{Gk}$

\section{INTRODUCTION}

A longstanding question is the origin of the non-zero quadrupole strength experimentally observed in the $\gamma^{\times} p \rightarrow$ $\Delta(1232)$ transition. The quadrupole transition occurs via the absorption of an $E 2$ or $C 2$ photon and typically requires non-zero orbital angular momentum in the $N$ or $\Delta$ wave function. This can arise from configuration mixing due to residual gluon or meson interactions between constituent quarks or from the photon directly coupling to the pion cloud. The latter is of particular importance at low $Q^{2}$ due to the strong $\Delta \rightarrow \pi N$ interaction, and numerous phenomenological reaction models have been developed to estimate the meson contribution to the transition current [1]. However until recently the data were not sufficiently accurate to test pion cloud calculations. New precision measurements of the $p\left(e, e^{\prime} p\right) \pi^{o}$ reaction at BATES, Jefferson Lab (JLAB) and MAMI have substantially improved our knowledge of the $Q^{2}$ dependence of the electric and Coulomb quadrupole/magnetic dipole ratios $R E M=E_{1+} / M_{1+}$ and $R S M=S_{1+} / M_{1+}$. In this paper we describe the JLAB/CLAS experiment and efforts to understand the model dependence of procedures used to extract the multipoles and resonance photocouplings.

\section{EXPERIMENT}

Previous CLAS electroproduction experiments in the $\Delta$ region at higher $Q^{2}$ were described in $[2,3,4]$. The new data were taken in Nov. 2002 using the CLAS spectrometer in Hall B at JLAB. An electron beam ( $E=1046 \pm 2 \mathrm{MeV}$, $I_{b}=10 \mathrm{nA}$ ) was incident on a tapered $2.0 \mathrm{~cm}$ long liquid hydrogen target cell with radius $R=0.3-0.7 \mathrm{~cm}$. Scattered electrons and hadrons were transported through a toroidal magnetic field and the CLAS detector packages. An energy threshold of $0.3 \mathrm{GeV}$ was set on the electron calorimeter analog energy sum, and the hardware trigger consisted of a coincidence of this signal and a Cerenkov signal in the same sector of CLAS. The resulting coverage in $Q^{2}$ and $W$ is shown in Figure 1 (left). Absolute normalization was verfied to within $3 \%$ using elastic scattering while the trigger efficiency was studied by comparing the inclusive $\left(e, e^{\prime}\right) W$ spectrum against parameterizations of previous measurements at SLAC and JLAB. After kinematic corrections the $W$ resolution was $7.5 \mathrm{MeV}$ in the lowest $Q^{2}$ bin (where $\int \vec{B} \cdot d \vec{l}$ is larg est.) Hadron detection efficiency was checked where possible using data from exclusive reactions. A GEANT simulation of CLAS was used to determine the acceptance, resolution and detection efficiency, where the physics event generator was based on a radiated version of the MADD model [5]. Both real and simulated data were binned using $6 Q^{2}, 15 W, 10 \cos \theta_{\pi}^{*}$ and $22 \phi_{\pi}^{*}$ bins. Final radiative corrections were performed using the EXCLURAD code developed at JLAB for exclusive reactions. Radiative tail contamination into the $\pi^{0}$ missing mass region from the elastic peak (Figure 1 (right)) was estimated from the GEANT simulation and otherwise removed using additional cuts on the laboratory angles differences $\theta_{p}-\theta_{\gamma^{*}}$ and $\phi_{e}-\phi_{p}$.

CP904, Shapes of Hadrons

edited by C. N. Papanicolas and A. M. Bernstein

(C) 2007 American Institute of Physics 978-0-7354-041 1-3/07/\$23.00 
Typical cross sections in the $p \pi^{0}$ center-of-mass (c.m.) system are shown in Figure 2 for invariant energy $W$ near the $\Delta(1232)$ peak. Also shown in these plots are Legendre polynomial fits discussed in the next section. For the lowest $Q^{2}$ some c.m. coverage was lost near $\phi_{\pi}^{*}=180^{\circ}$ due to the forward angle limit of the CLAS acceptance. This resulted in some reduction of sensitivity to the structure function $\sigma_{T L}$ which is proportional to $\cos \phi_{\pi}^{*}$.

\section{ANALYSIS}

Sensitivity to the small pion multipoles $E_{1+}$ and $S_{1+}$ occurs via their interference terms $\operatorname{Re}\left(E_{1+} M_{1+}^{*}\right)$ and $\operatorname{Re}\left(S_{1+} M_{1+}^{*}\right)$ with the dominant $M_{1+}$. These are extracted using the azimuthal and partial wave decomposition of the $p \pi^{0}$ c.m. cross section:

$$
\frac{1}{k} \frac{d \sigma}{d \Omega_{\pi}^{*}}=\sum_{\ell=0}^{2} A_{\ell}^{T+L} P_{\ell}\left(\cos \theta_{\pi}^{*}\right)+\sin ^{2} \theta_{\pi}^{*} C_{0}^{T T} \cos 2 \phi_{\pi}^{*}+\sin \theta_{\pi}^{*} \sum_{\ell=0}^{1} D_{\ell}^{T L} P_{\ell}\left(\cos \theta_{\pi}^{*}\right) \cos \phi_{\pi}^{*}
$$

where to first order, retaining only $M_{1+}$ dominated terms

$$
\begin{aligned}
\left|M_{1+}\right|^{2} & =A_{0}^{T+L} / 2, \\
\operatorname{Re}\left(E_{1+} M_{1+}^{*}\right) & =\left(A_{2}^{T+L}-2 C_{0}^{T T} / 3\right) / 8, \\
\operatorname{Re}\left(S_{1+} M_{1+}^{*}\right) & =D_{1}^{T L} / 6 .
\end{aligned}
$$

Here "T" and "L" refer to the transverse and longitudinal components of the virtual photon polarization and $k$ is a kinematic factor. By fitting the CLAS data with (1) the Legendre coefficients $A_{\ell}^{T+L}, C_{0}^{T T}$ and $D_{\ell}^{T L}$ were extracted as shown in Figure 3 for $Q^{2}=0.2 \mathrm{GeV}^{2}$. These coefficients clearly show a resonance-like $W$ dependence consistent with terms involving the $\Delta(1232)$ dominated $M_{1+}$. The accuracy of the truncated multipole expansion (TME) of (2-4) has been discussed at length by Kelly [6] for $Q^{2}=1.0 \mathrm{GeV}^{2}$. At much higher $Q^{2}$, where $M_{1+}$ dominance is no longer valid, the TME is a poor approximation and a model approach such as the Unitary Isobar Model (UIM) [8] must be used to separate the resonant and non-resonant multipoles. The UIM incorporates non-resonant $s-c h a n n e l$ Born and $t$-channel $\pi, \rho$ and $\omega$ exchange together with tails from higher resonances which are parameterized using fits to $W$ regions away from the $\Delta$ peak. All of these processes contribute to the strength of terms neglected in the TME. For the $Q^{2}$ range of this experiment, results for REM and $R S M$ from both the UIM fits and the TME equations (2-4) are shown in Table 1. At least some of the difference between the two approaches may arise from the fact that the UIM fit is constrained by the $W$ dependence of the data (see Figure 3 ), while the TME solution is at a single point $W=1.22 \mathrm{GeV}$ and subject more to statistical fluctuations. In any case, comparison of the model-independent TME solution to results coming from model analyses can provide an estimate of the magnitude of the background related truncation errors.

Figure 4 shows the $Q^{2}$ dependence of the magnetic dipole $G_{M}^{*}$, which is related to the isospin $I=3 / 2 \pi N$ multipole $\operatorname{Im}\left(M_{1+}^{3 / 2}\right)$ through the $\Delta$ width and a kinematic factor. The new exclusive electroproduction measurements of $G_{M}^{*}$ now extend over the range $Q^{2}=0.06-6.0 \mathrm{GeV}^{2}$, and confirm the rapid $Q^{2}$ falloff relative to the elastic dipole seen in previous inclusive measurements, but with much greater sensitivity to the resonant $M_{1+}$ multipole at higher $Q^{2}$. As previously noted, at least part of this rapid falloff may arise from the 'dressing' of the $\gamma^{*} N \rightarrow \Delta$ vertex by rescattering through the pion cloud. The 'bare' curve in Figure 4 was obtained in the Sato-Lee (SL) dynamical model [9] when the predicted 'dressed' $G_{M}^{*}$ form factor was fitted to data at $Q^{2}=0$ and to JLAB/Hall-C electroproduction data [10] at $Q^{2}=2.8,4.0 \mathrm{GeV}^{2}$. A comparison to the 'dressed' curve shows the increasing contribution from mesons as $Q^{2} \rightarrow 0$. Similar results have been obtained in the Dubna-Mainz-Taipei model [11].

The CLAS experimental quadrupole transition ratios $R E M$ and $R S M$ are shown in Figure 5 along with other recent experiments. The JLAB results are based on a full partial-wave analysis, while the MAMI and BATES data sets cover a more limited angle range. For the latter, MAID, DMT and SL model fits are used to estimate the $\Delta$ resonant multipoles. The other data points with errors are quenched lattice QCD calculations [12] which have undergone a linear chiral extrapolation to the physical pion mass. It is evident that while the calculations qualitatively account for the magnitude of $R E M$ and $R S M$, the $Q^{2}$ dependence is not consistently described. The low $Q^{2}$ underprediction of $R S M$ by LQCD is particularly striking. It was recently demonstrated, using a relativistic chiral effective field theory $(\chi \mathrm{EFT})$ calculation [13], that RSM may be particularly sensitive to a non-analytic dependence of the meson loop diagrams on the quark mass, rendering invalid the linear chiral extrapolation used in [12]. A strong negative slope near $Q^{2}=0$ is 
TABLE 1. Extracted values of $R E M=E_{1+} / M_{1+}$ and $R S M=S_{1+} / M_{1+}$ at $Q^{2}=0.16-0.36 \mathrm{GeV}^{2}$ from analysis of preliminary CLAS measurement of $p\left(e, e^{\prime} p\right) \pi^{0}$ reaction. Methods used are Truncated Multipole Expansion (TME), Unitary Isobar Model (UIM) and Sato-Lee dynamical model (SL). The SL3 column used an improved fit of the SL meson scattering potential model to $\pi N$ phase shifts. Errors are statistical only.

\begin{tabular}{|c|cccc|cccc|}
\hline \multirow{2}{*}{$Q^{2}$} & TME & UIM & SL & SL3 & TME & UIM & SL & SL3 \\
\hline 0.16 & $-1.94(0.36)$ & $-1.94(0.13)$ & $-2.45(0.2)$ & $-2.57(0.2)$ & $-4.88(0.36)$ & $-4.64(0.19)$ & $-4.44(0.35)$ & $-4.36(0.35)$ \\
0.20 & $-2.22(0.33)$ & $-1.68(0.18)$ & $-2.21(0.2)$ & $-2.31(0.2)$ & $-4.60(0.34)$ & $-4.62(0.18)$ & $-4.23(0.35)$ & $-4.14(0.35)$ \\
0.24 & $-1.91(0.39)$ & $-2.14(0.14)$ & $-2.70(0.2)$ & $-2.76(0.2)$ & $-5.02(0.39)$ & $-4.60(0.28)$ & $-4.32(0.35)$ & $-4.21(0.35)$ \\
0.28 & $-2.02(0.45)$ & $-1.69(0.27)$ & $-1.99(0.2)$ & $-2.07(0.2)$ & $-6.05(0.47)$ & $-5.50(0.31)$ & $-5.08(0.35)$ & $-4.97(0.35)$ \\
0.32 & $-2.71(0.54)$ & $-1.59(0.17)$ & $-2.29(0.2)$ & $-2.35(0.2)$ & $-5.97(0.54)$ & $-5.71(0.33)$ & $-4.87(0.35)$ & $-4.75(0.35)$ \\
0.36 & $-2.56(0.67)$ & $-1.52(0.27)$ & $-1.80(0.2)$ & $-1.82(0.2)$ & $-5.66(0.66)$ & $-5.79(0.43)$ & $-4.76(0.35)$ & $-4.56(0.35)$ \\
\hline
\end{tabular}

predicted by $\chi$ EFT for both REM and $R S M$ (see Figure 5). While for the lowest $Q^{2}$ the $R S M$ points from MAMI and BATES appear to agree with this prediction, both the MAMI and CLAS data at higher $Q^{2}$ are in better agreement with the SL and LQCD predictions. For REM, none of the new data show the strong $Q^{2}$ dependence predicted by $\chi \mathrm{EFT}$ near $Q^{2}=0$ although the data lie just outside of the estimated theoretical uncertainty arising from higher-order effects.

\section{BARE $\gamma^{*} N \rightarrow \Delta(1232)$ COUPLINGS}

Understanding the long-range pion cloud dynamics is necessary in order to access the more fundamental 'bare' $\gamma^{\times} N \rightarrow \Delta$ vertex. The bare vertex is presumably sensitive to the short-range interquark potential which is relevant to QCD-motivated models of resonance formation. Also the electromagnetic couplings $g_{M}, g_{E}$ and $g_{C}$ contain information about the quark core wave functions of the $N$ and $\Delta$. A frequently used parameterization of the $\gamma^{*} N \rightarrow \Delta$ vertex was given by Jones and Scadron [15]. These 'bare' couplings were determined in the SL dynamical model [14] by treating them as free parameters which were fitted to experimental photoproduction cross section and beam asymmetry data from LEGS and BONN. Their result was $g_{M}=1.9 \pm 0.05, g_{E}=0.0 \pm 0.025$ within the range of uncertainty $7<g_{\omega N N}<10.5$ of the $\omega$ meson coupling. In a subsequent paper [9] the authors fitted new data from Mainz, which favored the solution $g_{M}=1.85, g_{E}=0.025$ and $g_{\omega N N}=11.5$. The latest fit [18] to a much more extensive MAMI data set [17] has not appreciably changed the results of [9], aside from a small increase in $g_{E}$. As a result the dressed $E_{1+} / M_{1+}$ ratio is determined to be $R E M=-2.95 \pm 0.1 \%$.

This work was extended to electroproduction in [9] by fitting to the JLAB/HALL C data set [10] at $Q^{2}=$ 2.8,4.0 $\mathrm{GeV}^{2}$. Best results were obtained using the parameterization

$$
g_{x}\left(Q^{2}\right)=g_{x}(0)\left(\frac{1}{\left(1+Q^{2} / 0.71\right)^{2}}\right)^{2}\left(1+a Q^{2}\right) \exp \left(-b Q^{2}\right)
$$

where $x=M, E, C, a=0.154$ and $b=0.166$. The value of $g_{C}(0)$ was fixed using the long wavelength limit

$$
g_{C}(0)=-4 \frac{M_{\Delta}^{2}}{\left(M_{\Delta}^{2}-M_{N}^{2}\right)} g_{E}(0)
$$

Note that this ansatz assumes an identical $Q^{2}$ dependence for all three 'bare' electromagnetic couplings, and leads to the predictions for REM and $R S M$ corresponding to the 'dressed' $\gamma \sim N \rightarrow \Delta(1232)$ vertex, shown in Figure 5. At present it is not clear whether the difference between the experiments and the predicted $Q^{2}$ dependence reflects information about the bare couplings or about deficiencies in the model description of the $\pi N$ rescattering process, since the separation is somewhat model dependent. In any case within the SL model the bare couplings can be extracted in an unambiguous way by fitting to each individual $Q^{2}$ point, thus avoiding the use of (5). This was performed [18] for all recent data sets below $Q^{2}=2.0 \mathrm{GeV}^{2}$. Typical fits to recent BATES, CLAS and MAMI structure function data are shown in Figure 6, with the dashed line showing the SL prediction using (5). The extracted dressed ratios REM,RSM for the new CLAS data are listed in the Table 1 for two versions of the SL model. The SL 3 version used an improved fit of the meson scattering potential parameters to the $\pi N$ experimental phase shifts. Comparison of the SL and SL3 fits thus gives some idea of the 'internal' model dependence of the SL dynamical approach. Differences between the 
UIM and SL results may reflect more the absense of higher resonances in the SL approach, although this is still being studied.

The $Q^{2}$ dependence of the extracted bare electromagnetic couplings $g_{M}, g_{E}$ and $g_{C}$ are shown in Figure 7 . While $g_{M}$ mostly follows the parameterization given previously by (5), both $g_{E}$ and $g_{C}$ show a strong departure from smooth behavior at low $Q^{2}$. The simplest assumption, that the three couplings have an identical $Q^{2}$ dependence, is clearly ruled out by the data. The apparent rapid falloff in $Q^{2}$ of the bare quadrupole couplings near $Q^{2}=0$ could indicate the need for additional refinements to the SL dynamical model, or it could signify some additional long-range physics not yet incorporated. Given the expectation of strong chiral corrections to the lattice QCD result for RSM [13] and the large variations in the calculations shown in Figure 5, it is not yet clear what modifications to any of the models would be needed to describe the measured $Q^{2}$ evolution of REM and RSM. It is likely that more extensive measurements in the $Q^{2}=0.01-0.1 \mathrm{GeV}^{2}$ range would be useful. These should include polarization observables and more complete angular coverage, which will allow a more model-independent analysis.

\section{SUMMARY AND CONCLUSIONS}

New CLAS measurements of the $p\left(e, e^{\prime} p\right) \pi^{0}$ reaction at $Q^{2}=0.16-0.36 \mathrm{GeV}^{2}$ have been fitted with a Unitary Isobar Model and the Sato-Lee dynamical model in order to estimate the resonant $\gamma^{*} p \rightarrow \Delta(1232)$ quadrupole transition multipoles. The precision of the data, including model dependence in the extraction of multipoles, is approaching that which is necessary to test the latest chiral and dynamical meson cloud models. A comparison to the latest predictions shows qualitative agreement in magnitude, but the $Q^{2}$ evolution of the electric and scalar quadrupoles are not both described consistently by any models. Our new results from CLAS presented here in combination with recent measurements at BATES and MAMI suggest that there are some gaps in our understanding of how the pion cloud affects the electromagnetic coupling to the nucleon. The origin of the pion cloud and its association with the confinement and internal dynamics of the nucleon's consitituents is currently of much interest, for example, in the study of the nucleon's electric and magnetic polarizabilities. Another static property of the nucleon, the magnetic moment, is related to virtual $\Delta$ excitations via the GDH sum rule. Clearly the $\Delta(1232)$ resonance is an ideal system for studying the interaction between long- and short-range physics and should continue to serve as a benchmark for testing future calculations.

\section{ACKNOWLEDGMENTS}

This work was supported by the U.S. Department of Energy, Office of Nuclear Physics, under Contract No. DEFG02-96ER40950 and in part through Contract No. DE-AC05-060R23177, under which Jefferson Science Associates operates Jefferson Lab.

\section{REFERENCES}

1. V. Burkert V and T.-S. H. Lee, Int. J. of Mod. Phys. E13, 1035 (2004).

2. K. Joo, L.C. Smith et al. (CLAS Collaboration), Phys. Rev. Lett. 88, 122001 (2002).

3. H. Egiyan et al. (CLAS Collaboration), Phys. Rev. C73, 025204 (2006).

4. M. Ungaro et al. (CLAS Collaboration), Phys. Rev. Lett. 97, 112003 (2006).

5. L. Tiator et al., Eur. Jour. Phys. A19, 55 (2004).

6. J.J. Kelly, Phys. Rev. C 72, 048201 (2005).

7. J.J. Kelly et ai., Phys. Rev. Lett. 95, 102001 (2005).

8. I. Aznauryan. Phys. Rev. C 67, 015209 (2003).

9. T. Sato and T.-S. H. Lee, Phys. Rev. C63, 055201 (2001).

10. V. Frolov et al., Phys. Rev. Lett. 82, 45 (1999).

11. S. Kamalov and S. Yang, Phys. Rev. Lett. 83, 4494 (1999)

12. C. Alexandrou et al., Phys. Rev. C69, 114506 (2004); Phys. Rev. Lett. 94, 021601 (2005).

13. V. Pascalutsa and M. Vanderhaeghen, Phys. Rev. D73, 034003 (2006).

14. T. Sato and T.-S. H. Lee, Phys. Rev. C 54, 2660 (1996).

15. H.F. Jones and M.D. Scadron, Ann. of Phys. 81, 1 (1973).

16. D. Elsner el al., Eur. Phys. J. A 27, 91 (2006).

17. R. Leukel, Ph.D. Thesis, University of Mainz, (2001). 
18. B. Julia-Diaz, T.-S. H.Lee and L.C. Smith, in preparation.

19. S. Stave $e$ al., nucl-ex/0604013.

20. N. Sparveris, private communication and these proceedings.

21. C. Mertz et al., Phys. Rev. Lett. 86, 2963 (2001).

22. N. Sparveris et al., Phys. Rev. Lett 94, 022003 (2005). 

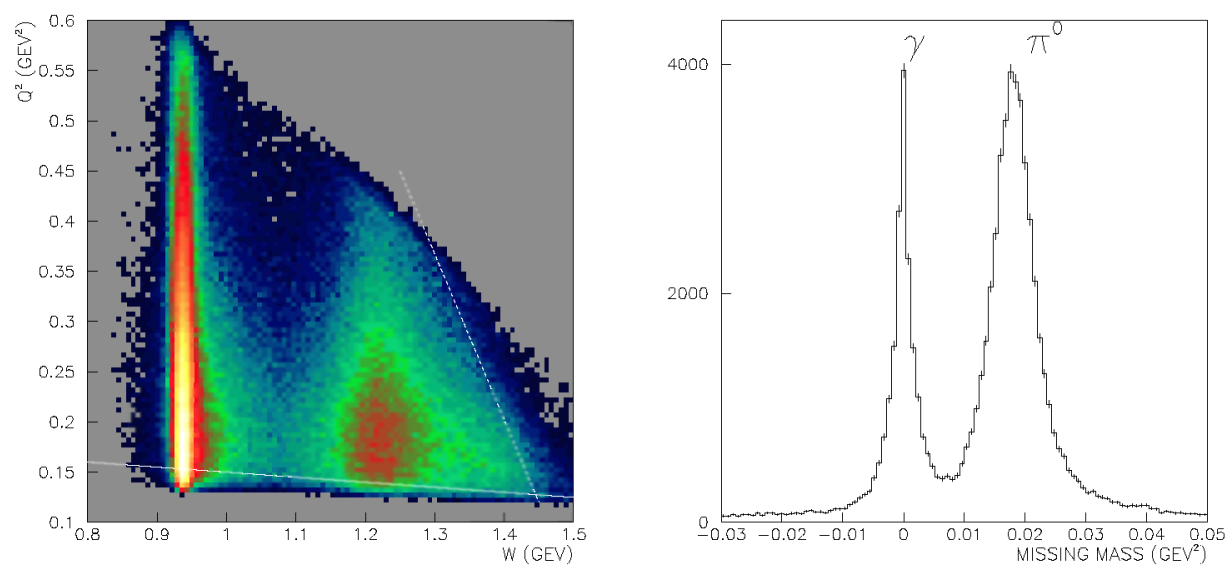

FIGURE 1. LEFT: Raw data from CLAS showing $\left(Q^{2}, W\right)$ coverage at beam energy $\mathrm{E}=1.046 \mathrm{GeV}$. Visible are the elastic peak at $W=0.938 \mathrm{GeV}$ and the $\Delta$ resonance at $W=1.23 \mathrm{GeV}$. Lines indicate the limits imposed by $\left(\theta_{e}, \phi_{e}\right)$ fiducial cuts (solid) and the scattered electron energy trigger threshold (dashed). RIGHT: Missing mass $M_{x}^{2}$ for $p\left(e, e^{\prime} p\right) X$ reaction for $1.1<W<1.2 \mathrm{GeV}$ and $Q^{2}<0.2 \mathrm{GeV}^{2}$.
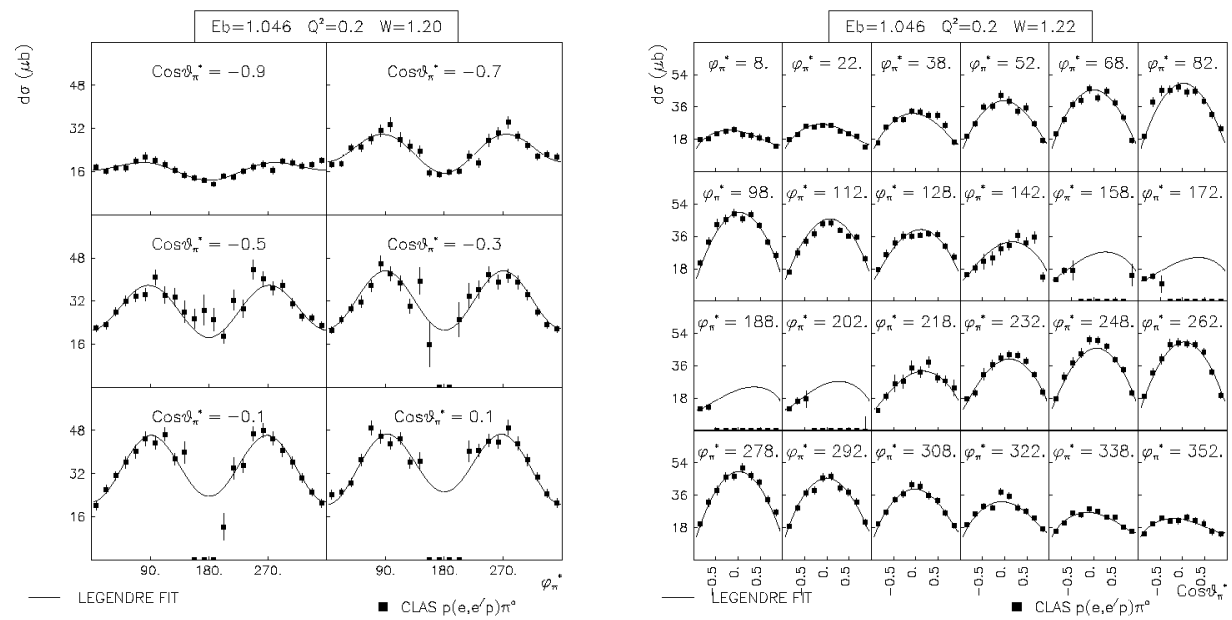

FIGURE 2. CLAS cross sections for the $p\left(e, e^{\prime} p\right) \pi^{0}$ reaction at $Q^{2}=0.2 \mathrm{GeV}^{2}$ and $W=1.20 \mathrm{GeV}$ as a function of $\phi_{\pi}^{*}$ (LEFT) and $\cos \theta_{\pi}^{s}$ (RIGHT). Solid line shows Legendre polynomial fit using Eq. 1 in text. 

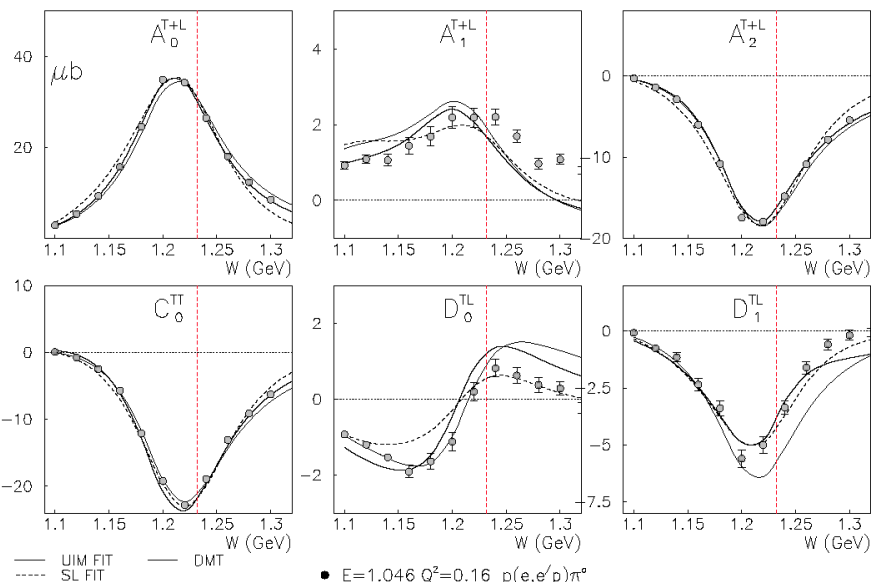

- $E=1.046 Q^{2}=0.16 p\left(e . e^{\prime} p\right) \pi^{0}$

FIGURE 3. Dependence on $W$ of partial wave coefficients from Legendre fit of experimental $\pi^{0}$ electroproduction structure functions $\sigma_{T+L}, \sigma_{T T}$ and $\sigma_{L T}$. Bold curves show results from model fits: UIM (solid), Sato-Lee (dashed). DMT curve is prediction from Dubna-Mainz-Taipei model [11]. Vertical line shows position of $\Lambda(1232)$ resonance. CLAS data taken at $\mathrm{E}=1.046 \mathrm{GeV}$ and $Q^{2}=0.16 \mathrm{GeV}^{2}$.

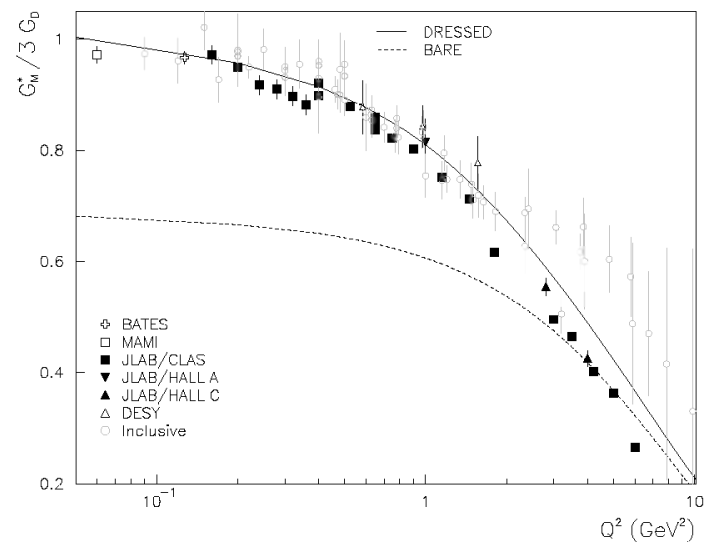

FIGURE 4. Magnetic dipole transition form factor $G_{M}^{*}$ for $\gamma^{*} N \rightarrow \Delta(1232)$, normalized to the elastic dipole $G_{d}$. Inclusive data are from pre-1990 experiments at Bonn, DESY and SLAC. Other data points were obtained from analyses of the exclusive $p\left(e, e^{\prime} p\right) \pi^{o}$ reaction. Curves from Sato-Lee model discussed in text. 

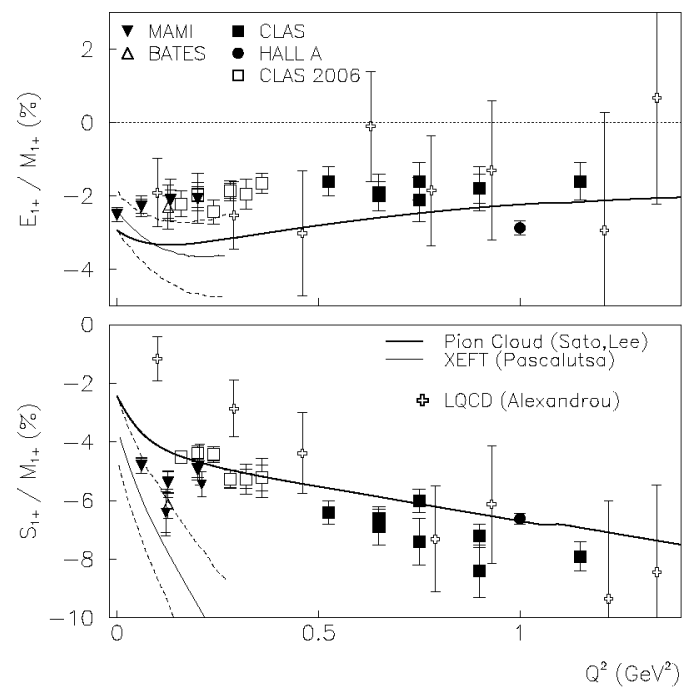

FIGURE 5. Quadrupole $\gamma^{*} N \rightarrow \Delta(1232)$ transition form factor ratios from current (CLAS 2006) and recent experiments [1]. Electric quadrupole $E_{1+}$ and scalar quadrupole $S_{1+}$ are normalized to the magnetic dipole $M_{1+}$. CLAS2006 points were obtained from average of UIM and SL model estimates in Table 1. Model error is included. Results from MAMI [19, 20, 16], BATES [22, 21] and JLAB/Hall A [7] are also included. Model calculations $[9,12,13]$ are discussed in the text. 

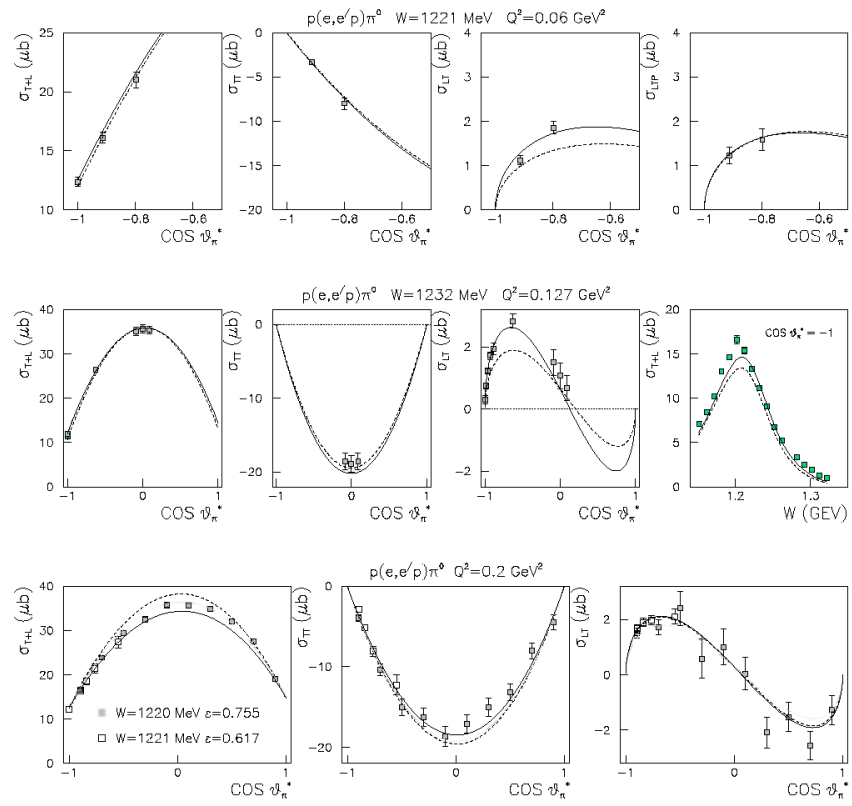

FIGURE 6. Fits of Sato-Lee dynamical model to experimental $p\left(e, e^{\prime} p\right) \pi^{0}$ structure functions. Solid lines show fit results, dashed line refers to the $Q^{2}$ parameterization of the 'bare' couplings $g_{M}, g_{E}$ and $g_{C}$ used in [9]. Data are from MAMI [19] at $Q^{2}=$ $0.06 \mathrm{GeV}^{2}\left(\right.$ top), BATES $[21,22]$ at $Q^{2}=0.127 \mathrm{GeV}^{2}$ (middle) and both CLAS $(W=1220 \mathrm{MeV})$ and MAMI $(W=1221 \mathrm{MeV})[20]$ at $Q^{2}=0.2 \mathrm{GeV}^{2}$ (bottom). 

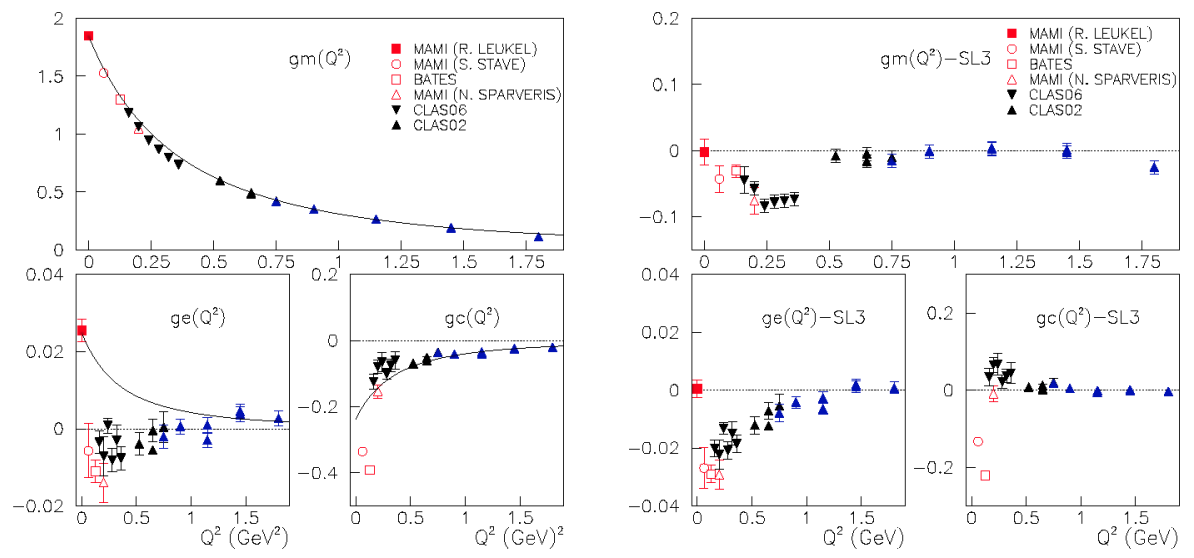

FIGURE 7. LEFT: Dependence on $Q^{2}$ of bare magnetic dipole, electric quadrupole and coulomb quadrupole couplings $g_{M}, g_{E}$ and $g_{C}$ for the $\gamma^{\prime \prime} p \rightarrow \Delta$ transition, extracted from fits of the SL dynamical model to BATES, CLAS and MAMI data. Solid line denotes the parameterization of Eq.(5) for the bare couplings used in the original SL model [9]. RIGHT: Same as left panel except couplings are shown relative to the SL parameterization $g_{x}\left(Q^{2}\right)$. For the SL3 solution $g_{M}(0)=1.848, g_{E}(0)=0.0255$ and $g_{C}(0)=-0.238$. 